\title{
The influence of prior knowledge on the retrieval- directed function of note taking in prior knowledge activation
}

Citation for published version (APA):

Wetzels, S., Kester, L., van Merrienboer, J. J. G., \& Broers, N. J. (2011). The influence of prior knowledge on the retrieval-directed function of note taking in prior knowledge activation. British Journal of Educational Psychology, 81(2), 274-291. https://doi.org/10.1348/000709910X517425

Document status and date:

Published: 01/06/2011

DOI:

10.1348/000709910X517425

Document Version:

Publisher's PDF, also known as Version of record

Document license:

Taverne

Please check the document version of this publication:

- A submitted manuscript is the version of the article upon submission and before peer-review. There can be important differences between the submitted version and the official published version of record.

People interested in the research are advised to contact the author for the final version of the publication, or visit the DOI to the publisher's website.

- The final author version and the galley proof are versions of the publication after peer review.

- The final published version features the final layout of the paper including the volume, issue and page numbers.

Link to publication

\footnotetext{
General rights rights.

- You may freely distribute the URL identifying the publication in the public portal. please follow below link for the End User Agreement:

www.umlib.nl/taverne-license

Take down policy

If you believe that this document breaches copyright please contact us at:

repository@maastrichtuniversity.nl

providing details and we will investigate your claim.
}

Copyright and moral rights for the publications made accessible in the public portal are retained by the authors and/or other copyright owners and it is a condition of accessing publications that users recognise and abide by the legal requirements associated with these

- Users may download and print one copy of any publication from the public portal for the purpose of private study or research.

- You may not further distribute the material or use it for any profit-making activity or commercial gain

If the publication is distributed under the terms of Article $25 \mathrm{fa}$ of the Dutch Copyright Act, indicated by the "Taverne" license above, 


\title{
The influence of prior knowledge on the retrieval-directed function of note taking in prior knowledge activation
}

\author{
Sandra A. J. Wetzels ${ }^{\prime *}$, Liesbeth Kester', \\ Jeroen J. G. van Merriënboer ${ }^{12}$ and Nick J. Broers ${ }^{3}$ \\ 'Centre for Learning Sciences and Technologies/Netherlands Laboratory \\ for Lifelong Learning, Open Universiteit in the Netherlands, Heerlen, The \\ Netherlands \\ ${ }^{2}$ School of Health Professions Education, Maastricht University, \\ The Netherlands \\ ${ }^{3}$ Department of Methodology and Statistics, Maastricht University, \\ The Netherlands
}

Background. Prior knowledge activation facilitates learning. Note taking during prior knowledge activation (i.e., note taking directed at retrieving information from memory) might facilitate the activation process by enabling learners to build an external representation of their prior knowledge. However, taking notes might be less effective in supporting prior knowledge activation if available prior knowledge is limited.

Aims. This study investigates the effects of the retrieval-directed function of note taking depending on learners' level of prior knowledge. It is hypothesized that the effectiveness of note taking is influenced by the amount of prior knowledge learners already possess.

Sample. Sixty-one high school students participated in this study. A prior knowledge test was used to ascertain differences in level of prior knowledge and assign participants to a low or a high prior knowledge group.

Method. A $2 \times 2$ factorial design was used to investigate the effects of note taking during prior knowledge activation (yes, no) depending on learners' level of prior knowledge (low, high) on mental effort, performance, and mental efficiency.

Results. Note taking during prior knowledge activation lowered mental effort and increased mental efficiency for high prior knowledge learners. For low prior knowledge learners, note taking had the opposite effect on mental effort and mental efficiency.

\footnotetext{
* Correspondence should be addressed to Sandra A. J. Wetzels, Centre for Learning Sciences and Technologies/Netherlands Laboratory for Lifelong Learning, Open Universiteit in the Netherlands, PO Box 2960, 640I DL Heerlen, The Netherlands (e-mail: sandra.wetzels@ou.nl).
} 
Conclusions. The effects of the retrieval-directed function of note taking are influenced by learners' level of prior knowledge. Learners with high prior knowledge benefit from taking notes while activating prior knowledge, whereas note taking has no beneficial effects for learners with limited prior knowledge.

Prior knowledge activation has a strong positive impact on learning. Schmidt (1982), for example, activated students' prior knowledge by means of problem analysis. Students were asked to explain the problem of osmosis (i.e., swelling and shrinking of a red blood cell in pure water and a salt solution) in terms of an underlying process, principle, or mechanism. When formulating hypotheses regarding the problem of osmosis, students relied on their prior knowledge to account for the observed phenomena. Students who activated prior knowledge showed superior recall and transfer as compared to students who did not activate prior knowledge. Prior knowledge activation - in this case by means of problem analysis - functioned as a bridge between existing prior knowledge and knowledge still to be acquired. This facilitated the integration of new knowledge into already existing knowledge structures resulting in higher recall and better understanding of new information.

The present study explores the effects of note taking during prior knowledge activation. More specifically, it is investigated if and how note taking during prior knowledge activation influences the activation process and learning, taking learners' levels of prior knowledge into account. The structure of this introduction is as follows. First, the facilitative effects of prior knowledge activation on learning are described. Second, traditional note taking research is shortly described and the differences with note taking as operationalized in the present study are emphasized. Finally, it is hypothesized how note taking during prior knowledge activation may facilitate the activation process and learning, and how the effects of note taking are influenced by learners' levels of prior knowledge.

\section{Prior knowledge activation}

In line with Schmidt (1982), many studies have provided evidence for the facilitative effects of prior knowledge activation on learning (e.g., Chi, de Leeuw, Chiu, \& LaVancher, 1994; De Grave, Schmidt, \& Boshuizen, 2001; Goetz, Schallert, Reynolds, \& Radin, 1983; Machiels-Bongaerts, Schmidt, \& Boshuizen, 1995). Prior knowledge activation involves the transfer of available knowledge from long-term memory to working memory. If new, potentially meaningful information is related to the assimilative context of existing knowledge held in working memory, this information can be integrated in the existing knowledge base. This results in the elaboration and refinement of the existing knowledge base, with beneficial effects on recall and understanding (Mayer, 1979). So, the availability of prior knowledge is not sufficient to achieve higher learning outcomes. This knowledge should be retrieved and activated in order to establish relationships between existing knowledge and new information.

The way in which knowledge is represented in memory influences how easily, accurately, and efficiently knowledge can be retrieved and activated. Important units of knowledge are concepts. A concept is a general idea about an object (e.g., apple) constructed by means of abstraction that is related to other concepts (e.g., fruit, banana, green). According to network models, concepts are stored as nodes with links between nodes representing relations between concepts. This interconnected pattern of nodes 
enables learners to meaningfully organize knowledge contained in the connections among the various nodes. If prior knowledge is activated, specific nodes in the network are activated. This activation can easily spread to other connected nodes. The more often particular links between nodes are used, the stronger these links become. As a result of frequent use, learning takes place through strengthening of connections. In addition, the network provides a framework in which new information can be integrated. This framework facilitates learning because it offers the opportunity to establish connections between new information and previous experiences (i.e., nodes). The richer the integrated framework, the higher the chance that new information can be connected to already existing knowledge, enhancing both recall and transfer of knowledge (Anderson, 1983).

\section{Prior knowledge activation strategies}

There are different strategies that can be used to activate learners' prior knowledge. Problem analysis, for example, is a strategy in which learners collaboratively activate their prior knowledge when constructing explanations for a presented problem (e.g., De Grave et al., 2001; Schmidt, 1982). This is comparable to self-explanation in which learners generate explanations to themselves using their prior knowledge (Chi et al., 1994). Another strategy that is frequently used in prior knowledge activation is mobilization where learners are explicitly encouraged to bring to mind all knowledge they have in a certain domain (Peeck, 1982). Mobilization is considered a bottomup strategy; it serves a broad stage-setting function that provides learners with a relevant context in which new information can be integrated (Peeck, van den Bosch, \& Kreupeling, 1982). Because of this bottom-up stage-setting function, mobilization is a useful strategy for learners regardless of their level of prior knowledge.

Machiels-Bongaerts et al. (1995) asked students to mobilize all knowledge they had about the fishery policy of the European Union and its consequences. A control group activated prior knowledge about a neutral topic. Subsequently, all students studied a text describing the effects of the restrictive EU fishery policy on a small imaginary fishermen's village. The text contained information about the background of the fishery policy and its consequences (e.g., a rise in unemployment) that were expected to correspond to the activated prior knowledge of the experimental group. Furthermore, the text contained new information (e.g., an alternative income source) that became important in light of the activated prior knowledge. The experimental group outperformed the control group in recall of information from the text. This higher recall was caused by enhanced recall of both the information that was explicitly activated and the newly presented information. By establishing relations between the activated prior knowledge and the new information, the integration of the new information into the existing knowledge base is facilitated. So, mobilization enables learners to bridge the gap between their prior knowledge and new information provided to them.

Before outlining the potentially beneficial role of note taking in prior knowledge activation, the next section describes the focus of traditional note taking research and emphasizes the differences with note taking as operationalized in the present study.

\section{Note taking}

In traditional note taking research, note taking is mainly perceived as a data collection procedure aimed at encoding and storing information for examination purposes. 
Research focused primarily on learning from taking notes while attending a lecture (e.g., Austin, Gilbert Lee, Thibeault, Carr, \& Bailey, 2002; Kiewra et al., 1991) or reading a text (e.g., Kobayashi, 2009; Slotte \& Lonka, 1999). The encoding function of note taking signifies that the process of taking notes while attending a lecture or reading a text is beneficial for learning (Di Vesta \& Gray, 1972). This effect is determined by comparing learning outcomes (e.g., recall, academic achievement) of learners who were allowed to take (but not review) notes while acquiring new knowledge with learning outcomes of learners who were not allowed to take notes. So, the encoding effect represents the effects of note taking during learning. Most studies have shown that learners who take notes reach higher learning outcomes than learners who do not take notes (e.g., Barnett, Di Vesta, \& Rogozinski, 1981). Note taking can be used to organize information and establish personally relevant relations which make a lecture or text more comprehensible, facilitating learning.

Overall, the encoding effect of note taking is supported (cf. Kiewra, 1985; Kobayashi, 2005). However, some studies could not support the notion that taking notes is beneficial for learning. For example, Kobayashi (2009) and Peper and Mayer (1978, 1986) found that taking notes did not result in higher learning outcomes than not taking notes. These inconsistent findings may be related to the quality of the note taking procedures. According to Peper and Mayer (1978, 1986), note taking enhances learning if learners actively process the learning materials and relate it to their prior knowledge. However, learners often take notes that represent a verbatim account of the learning materials in which no personally relevant relations are established. In these situations, note takers may not show higher learning outcomes than learners who did not take notes.

In addition to the encoding function, note taking has traditionally been assigned an external storage function that indicates that having notes available for review after attending a lecture or reading a text is beneficial for learning (Di Vesta \& Gray, 1972). The external storage function is determined by comparing learning outcomes of learners who reviewed their notes with learning outcomes of learners who did not review their notes. So, the external storage function represents the effects of note taking after learning. Overall, the external storage function of note taking is supported. Kiewra (1985) analysed 22 studies of which 17 studies supported the external storage function; learners who reviewed their notes reached higher learning outcomes than learners who did not review their notes.

In this study, the effects of note taking are investigated in a context that differs from traditional note taking research in several respects. First, the effects of note taking are not investigated in the context of learning from a lecture or a text but in the context of prior knowledge activation. More specifically, it is investigated if and how note taking may support the activation process and subsequent learning. Second, note taking is not assumed to serve an encoding or external storage function. Prior knowledge is already encoded in long-term memory and therefore, note taking has no encoding function. In addition, learners are not given an opportunity to review their notes, so note taking also does not have an external storage function. In this study, note taking only serves a retrieval-directed function. By means of taking notes, the retrieval of information from long-term memory to working memory is facilitated. Third, traditional note taking research studied the effects of note taking during and/or after learning. In contrast, in this study, the effects of note taking before learning are investigated. Learners take notes 
to externally represent their prior knowledge before they are provided with learning materials.

\section{The retrieval-directed function of note taking}

How can note taking be used to support retrieval during prior knowledge activation? When considering the effects of prior knowledge activation, working memory is an important factor. Learners can hold about seven elements at a time in working memory (Baddeley, 1992). When required to simultaneously process elements, the capacity of working memory is even more severely limited to about three elements (Van Merriënboer \& Sweller, 2005). This implies that there are limits on the amount of information (i.e., the number of nodes) that can be simultaneously activated and processed. Learners might be overwhelmed by the activation process leading them to experience cognitive overload. If learners are overloaded, the activation process will be hampered because there is not enough capacity to activate all elements in the existing knowledge base (Van Merriënboer \& Sweller, 2005). Cognitive overload might be prevented if learners build an external representation of their prior knowledge by taking notes. When note taking is used this way, it serves a retrieval-directed function because, aided by the notes, learners can retrieve and thus activate more concepts than they could do without the notes. In addition, learners can relate concepts to one another and thereby create an organizing framework of their prior knowledge without having to keep all concepts active in working memory. This facilitates the activation process by reducing the load imposed on working memory during prior knowledge activation. When confronted with new materials, learners can hold the (re)organized framework in working memory and use it to integrate new material into the framework, which facilitates learning.

The retrieval-directed function of note taking is expected to facilitate the process of prior knowledge activation by reducing the load imposed on working memory. However, the supporting effects of note taking might be influenced by learners' levels of prior knowledge. Taking notes is assumed to be less effective if learners have only limited relevant prior knowledge in a domain (i.e., material is unfamiliar). For these learners, knowledge is not meaningfully organized, because their knowledge is not yet represented in an interconnected pattern of nodes (Anderson, 1983). This makes it difficult for them to build a coherent external representation by taking notes, because they cannot distinguish relevant from irrelevant concepts or draw relations between concepts (Anderson, 1977). Therefore, note taking is not expected to have beneficial offloading effects on working memory for learners who have only limited prior knowledge in a certain domain.

In sum, the main goal of this study is to investigate the effects of retrieval-directed note taking in prior knowledge activation for learners with different levels of prior knowledge. It is hypothesized that the effectiveness of note taking is influenced by the amount of prior knowledge learners already possess in a certain domain (i.e., biology). Learners with relatively high prior knowledge are expected to benefit from retrieval-directed note taking because externally representing their prior knowledge by means of taking notes will reduce the load imposed on working memory. This facilitates the activation process and thereby learning. In contrast, retrieval-directed note taking is not expected to have any positive effects for learners with relatively low prior knowledge. These learners are not able to build a coherent external representation of their prior knowledge and will 
therefore not benefit from the potentially beneficial offloading effects of taking notes on working memory.

\section{Method}

\section{Participants}

Sixty-one students in 11th grade (34 males and 27 females; mean age $=17.07$ years, $S D=0.75)$ participated in this study. To guarantee differences in level of prior knowledge concerning the circulatory system, students with and without biology in their final exams were asked to participate. A $2 \times 2$ factorial design was used to investigate the retrievaldirected function of note taking depending on learners' levels of prior knowledge. All participants completed a prior knowledge test about the circulatory system (see 'Materials'). The maximum score was $12(M=5.77, S D=3.08)$. Participants were assigned to a low prior knowledge or a high prior knowledge group based on the median score (value ' 5 ') of the prior knowledge test. Participants who scored below or at the median were assigned to the low prior knowledge group $(N=37)$ and participants who scored above the median were assigned to the high prior knowledge group $(N=$ 24). Thus, the terms 'low prior knowledge' and 'high prior knowledge' were used in a relative sense. To test if the two prior knowledge groups differed in their level of prior knowledge concerning the circulatory system, a $t$ test was carried out. The low prior knowledge group $(M=3.65, S D=1.21)$ scored significantly lower on the prior knowledge test than the high prior knowledge group $(M=9.04, S D=2.03), t(33.61)=$ $-11.730, p<.001$, Cohen's $d=3.23$. Subsequently, participants were randomly assigned to two note taking conditions resulting in the following four experimental conditions: low prior knowledge/no-note taking $(N=17)$, low prior knowledge/note taking $(N=$ $20)$, high prior knowledge/no-note taking $(N=13)$, and high prior knowledge/note taking $(N=11)$.

\section{Materials}

Prior knowledge test

The prior knowledge test about the circulatory system was a paper-and-pencil test administered to ascertain differences in level of prior knowledge and assign participants to the low prior knowledge or the high prior knowledge group. It contained 12 multiple choice items with 4 answer options that measured knowledge and understanding of the circulatory system. Six items assessed participants' prior knowledge of the structure of the circulatory system (e.g., through which blood vessels does blood flow from the leg to the heart?) and six items assessed knowledge of the functioning of the heart (e.g., what is the function of the heart valves?). One point was given for each correctly answered item on the prior knowledge test resulting in a maximum score of 12 . Reliability of the prior knowledge test was $\alpha=.77$ (Cronbach's alpha).

Prior knowledge activation pictures

To initiate prior knowledge activation, pictures illustrating the structure of the circulatory system and the functioning of the heart were presented. The picture used to activate participants' prior knowledge about the structure of the circulatory system is presented in Figure 1. 


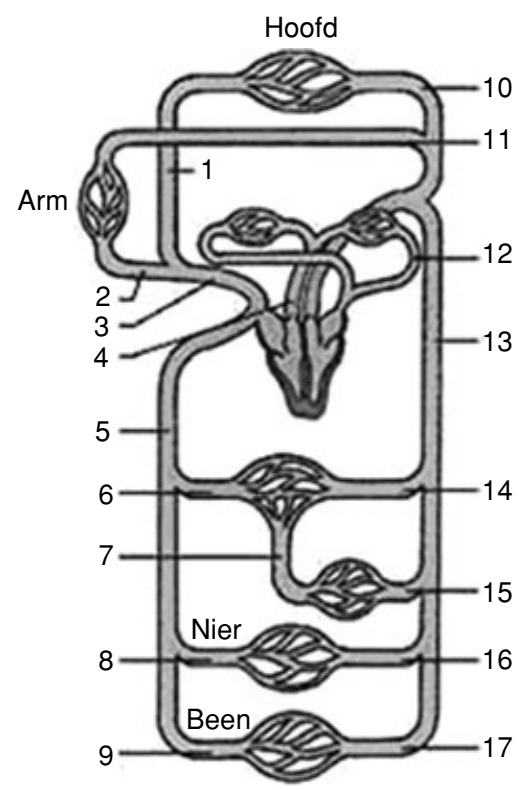

Figure I. Picture used to activate prior knowledge about the structure of the circulatory system.

\section{Learning tasks}

Twelve learning tasks were presented in the learning phase. These learning tasks were organized in two sequences of six tasks with one sequence focusing on the structure of the circulatory system, and the other sequence on the functioning of the heart. A sequence of learning tasks started with a worked-out example that contained the main principles necessary to solve the problems in the task sequence. For example, the worked-out example in the task sequence about the structure of the circulatory system described in detail how blood flows through the body. After the worked-out example with high build-in support, three tasks with diminishing learner support were presented. Learner support was provided by using so-called reversal and completion problems. In a reversal problem, the correct solution was already given encouraging backward reasoning of why this solution fitted the problem posed. In addition, two types of completion problems were used. In the first type, part of the solution was given and had to be completed by the participants to solve the problem. In the second type, all solution steps were given in a random order and had to be rearranged to solve the problem. These two types of completion problems and the correct solution are illustrated in the Appendix. The last tasks in the sequence were two conventional problems (i.e., no solution steps were provided).

\section{Transfer tasks}

Twelve transfer tasks assessed participants' knowledge and understanding of both the structure of the circulatory system and the functioning of the heart. They differed from each other on a continuum from near to far transfer. For near transfer tasks, learned principles were applied in familiar situations. The underlying structure did not differ from the structure of learning tasks, but these tasks contained different surface features, which were irrelevant for solving the tasks. For example, participants were asked to 
describe how a blood clot originating in the brain could get stuck in a leg. For far transfer tasks, learned principles had to be applied in new, unfamiliar situations. The structural features of these tasks were more or less different from those of the learning tasks. For example, participants were presented with a problem concerning a child with a congenital heart defect in which the left ventricle was underdeveloped. After an operation, the vena cava were directly connected to the lung arteries and the aorta was connected to the right ventricle. When describing how blood flows from the liver to the brain, participants had to take into account the child's abnormal blood flow.

\section{Scoring}

Time-on-task

Time to solve problems was automatically recorded for each learning and transfer task.

\section{Mental effort}

Mental effort was measured using the mental effort rating scale of Paas (1992). This measure was used to assess how much mental effort participants had to invest to complete the prior knowledge test, activate their prior knowledge, and solve each learning and transfer task. Mental effort was rated on a nine-point scale ranging from 'very, very little effort' (1) to 'very, very much effort' (9).

\section{Performance}

For each learning and transfer task, partial credits were given for a correct solution step or answer leading to a maximum score of 1 for each task.

\section{Mental efficiency}

Efficiency $(E)$ was calculated by relating transfer test performance and invested mental effort during the transfer test using the formula of Paas and van Merriënboer (1993). Performance and mental effort scores were first standardized, and then the $z$ scores were entered into the formula:

$$
E=\frac{Z_{\text {performance }}-Z_{\text {mental effort }}}{\sqrt{ } 2} .
$$

High efficiency indicated a transfer test performance that was higher than might be expected on the basis of invested mental effort during the transfer test, while a low efficiency indicated a transfer test performance that was lower than might be expected on the basis of invested mental effort. For example, the instructional procedure resulted in a higher mental efficiency for participants who attained a certain performance level with a minimum investment of mental effort than for participants who attained the same performance with a maximum investment of mental effort.

\section{Activated knowledge}

Activated knowledge was measured by analysing the notes taken by the note taking groups and think-aloud protocols recorded in a randomly selected subset $(N=$ 22) of all participants. The protocols were registered using Audacity version 1.2.6 
(http://audacity.sourceforge.net) and a headset. These protocols provided information on the knowledge that was actually activated by the learners. For determining correctness and relevancy of activated prior knowledge, notes and think-aloud protocols were scored according to a coding scheme that contained all important concepts necessary for solving learning and transfer tasks. The worked-out examples were used to distinguish these important concepts (e.g., left ventricle, sinus node) and relations between these concepts (e.g., blood flows from the right ventricle to the lung artery, electrical activity spreads from the sinus node to the atrioventricular node). Two independent raters scored both the number of concepts, the number of relations between these concepts, and the number of correct relations using the coding scheme. Inter-rater reliability was $r=.997$ (intra-class correlation).

\section{Procedure}

To avoid potential interference with prior knowledge activation, the prior knowledge test was administered more than 5 days before the experiment. In the experimental session, prior knowledge was activated through mobilization initiated by the prior knowledge activation pictures. First, the picture of the structure of the circulatory system was presented on a computer screen for $5 \mathrm{~min}$ and all participants were given the following instructions: 'Bring to mind everything you know about the way blood flows through the body'. Think-aloud protocols were recorded for a subset of all participants. During mobilization, all participants were also given this picture on a piece of paper. Only participants in the note taking conditions were instructed to take notes while mobilizing using the paper version of the picture. Participants in the no-note taking conditions were not given an instruction to take notes. It was closely observed whether participants followed the note taking instructions. Second, after mobilization, all participants were asked to hand in the paper version of the picture to prevent participants in the note taking conditions from using their notes while working on the tasks. Then, all participants studied the worked-out example about the structure of the circulatory system and performed the remaining learning tasks concerning this aspect of the circulatory system.

After mobilizing prior knowledge and working on tasks about the structure of the circulatory system, participants started working on the sequence of tasks about the functioning of the heart. This sequence zoomed in on a particular aspect of the circulatory system and required more elaborated knowledge than the preceding task sequence about the structure of the circulatory system. Therefore, prior knowledge was again mobilized before participants worked on these tasks. Participants were provided with the picture illustrating the functioning of the heart and were instructed to ' . . . bring to mind everything you know about the electrical system and the functioning of the heart'. Again, all participants were given a paper version of the picture. Participants in the note taking conditions were instructed to take notes while mobilizing using the paper version of the picture. After mobilization, participants were again asked to hand in this paper version. Then, participants studied the worked-out example about the functioning of the heart and performed the remaining learning tasks concerning this aspect of the circulatory system.

After all learning tasks were performed, transfer tasks were presented. Participants in the note taking conditions were not allowed to review or otherwise use their notes while working on the learning and transfer tasks. All participants were instructed not to take notes while performing the learning and transfer tasks. 
Participants rated the amount of mental effort they had to invest (a) after completing the prior knowledge test, (b) after mobilizing prior knowledge about the structure of the circulatory system, (c) after mobilizing prior knowledge about the functioning of the heart, (d) after each learning task, and (e) after each transfer task. All tasks and mental effort scales were presented on a computer screen. Participants were allowed to work on the learning and transfer tasks at their own pace.

\section{Results}

\section{Time-on-task}

For all statistical tests, a significance level of .05 was maintained. Table 1 provides an overview of the means and standard deviations of time-on-task and the dependent variables mental effort, performance, and mental efficiency.

Table I. Means and standard deviations of time-on-task, mental effort, performance, and mental efficiency

\begin{tabular}{|c|c|c|c|c|c|c|c|c|}
\hline & \multicolumn{4}{|c|}{ Low prior knowledge } & \multicolumn{4}{|c|}{ High prior knowledge } \\
\hline & \multicolumn{2}{|c|}{ No notes } & \multicolumn{2}{|c|}{ Notes } & \multicolumn{2}{|c|}{ No notes } & \multicolumn{2}{|c|}{ Notes } \\
\hline & M & $S D$ & $M$ & $S D$ & M & $S D$ & M & $S D$ \\
\hline Time-on-task learning phase (s) & III & 44 & 111 & 23 & 123 & 39 & 150 & 38 \\
\hline Time-on-task transfer phase (s) & 81 & 24 & 89 & 17 & 97 & 33 & 111 & 27 \\
\hline Mental effort learning phase & 5.79 & 1.16 & 6.28 & 1.29 & 5.02 & 1.49 & 4.42 & I.II \\
\hline Mental effort transfer phase & 5.61 & 1.49 & 6.59 & 1.26 & 5.42 & 1.30 & 4.65 & 1.28 \\
\hline Performance learning phase & 3.95 & 1.99 & 4.14 & 2.16 & 6.04 & 1.86 & 7.32 & 1.65 \\
\hline Performance transfer phase & 3.49 & 1.43 & 3.85 & 1.50 & 5.61 & 1.78 & 6.60 & 1.60 \\
\hline Mental efficiency transfer phase & -0.29 & 0.90 & -0.62 & 0.90 & 0.56 & 1.10 & 1.27 & 0.85 \\
\hline
\end{tabular}

To control for potentially confounding effects of time-on-task, time to perform learning and transfer tasks was explored first. A main effect of prior knowledge on time-on-task was found for the learning phase $(F(1,58)=6.939$, MSE $=9,205.372, p<$ $\left..05, \eta_{\mathrm{p}}^{2}=.107\right)$ and the transfer phase $(F(1,58)=8.324, \operatorname{MSE}=5,078.609, p<.01$, $\left.\eta_{\mathrm{p}}^{2}=.126\right)$. The high prior knowledge group spent more time working on learning and transfer tasks than the low prior knowledge group. No other main effects or interaction effects were found.

For subsequent analyses, data were analysed by means of 2 (low prior knowledge vs. high prior knowledge) $\times 2$ (no-note taking vs. note taking) ANCOVAs with time-on-task as a covariate. For analysing learning task results, time-on-task during the learning phase was used as a covariate. For analysing transfer task results, both time-on-task during the learning phase and time-on-task during the transfer phase were used as covariates.

\section{Mental effort}

An interaction effect between level of prior knowledge and note taking was found for mental effort invested during the transfer phase $(F(1,55)=6.688$, MSE $=12.149$, 


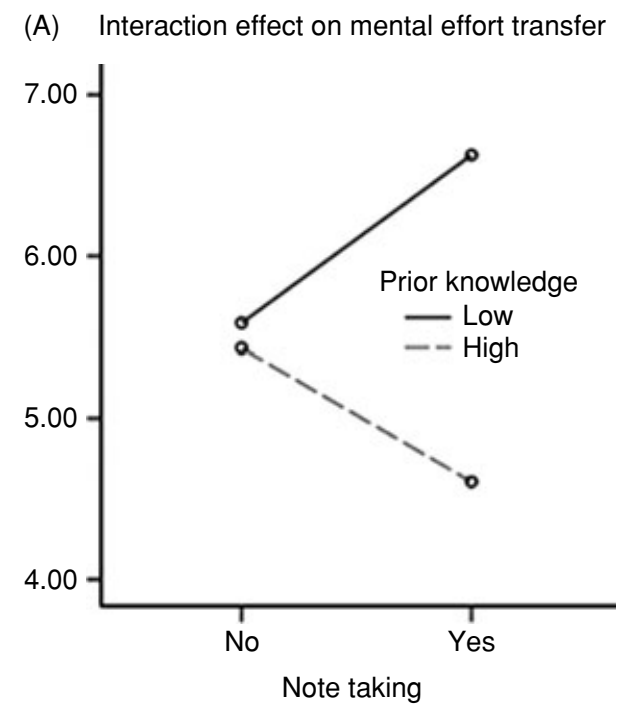

(B) Interaction effect on mental efficiency transfer

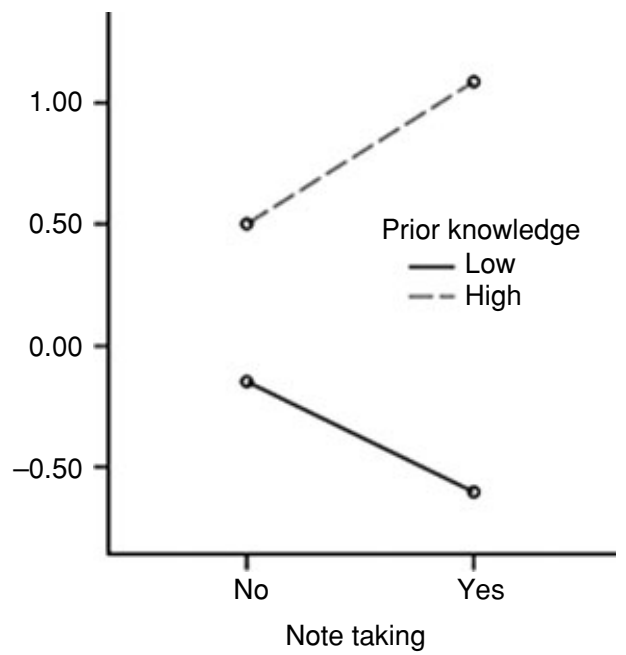

Figure 2. Interaction effect between level of prior knowledge and note taking for mental effort (A) and mental efficiency (B) during transfer.

$p<.05, \eta_{\mathrm{p}}^{2}=.108$ ). For high prior knowledge learners, note taking yielded a decrease in mental effort invested while working on transfer tasks, whereas note taking yielded the opposite effect on mental effort for low prior knowledge learners (see Figure 2A). Post hoc analyses revealed that mental effort of note takers was influenced by their level of prior knowledge. More specifically, mental effort of note takers with low prior knowledge was higher than mental effort of note takers with high prior knowledge $\left(F(1,55)=13.662\right.$, MSE $\left.=24.818, p<.01, \eta_{p}^{2}=.199\right)$. Moreover, note taking increased mental effort for low prior knowledge learners as compared to low prior knowledge learners who did not take notes $(F(1,55)=5.367$, MSE $=9.751, p<.05$, $\left.\eta_{\mathrm{p}}^{2}=.089\right)$.

In addition to the interaction effect between level of prior knowledge and note taking, a main effect of prior knowledge was found for mental effort invested during the learning phase $\left(F(1,57)=12.170\right.$, MSE $\left.=20.572, p<.01, \eta_{p}^{2}=.176\right)$ and the transfer phase $\left(F(1,55)=7.945, \mathrm{MSE}=14.433, p<.01, \eta_{\mathrm{p}}^{2}=.126\right)$. The high prior knowledge group invested less mental effort while working on learning tasks and transfer tasks than the low prior knowledge group (see Table 1). No other significant effects were found.

\section{Performance}

A main effect of prior knowledge on performance was found for both learning tasks $\left(F(1,57)=17.235\right.$, MSE $\left.=60.001, p<.001, \eta_{\mathrm{p}}^{2}=.232\right)$ and transfer tasks $(F(1,56)=$ 22.789, MSE $\left.=41.164, p<.001, \eta_{p}^{2}=.289\right)$. As Table 1 shows, the high prior knowledge group performed higher on learning tasks and transfer tasks than the low prior knowledge group. No other significant effects were found. There was no interaction effect between level of prior knowledge and note taking on learning task performance $\left(F(1,56)=0.355\right.$, MSE $\left.=1.252, \mathrm{~ns}, \eta_{\mathrm{p}}^{2}=.006\right)$ or transfer test performance $\left(F(1,55)=0.408\right.$, MSE $\left.=.746, \mathrm{~ns}, \eta_{\mathrm{p}}^{2}=.007\right)$. Furthermore, although note takers 
performed higher than participants who did not take notes (see Table 1), this difference did not reach statistical significance.

\section{Mental efficiency}

An interaction effect between level of prior knowledge and note taking was found for mental efficiency during the transfer phase $(F(1,55)=4.786$, MSE $=3.799, p<.05$, $\left.\eta_{\mathrm{p}}^{2}=.08\right)$. For high prior knowledge learners, note taking yielded an increase in mental efficiency during the transfer phase, whereas note taking yielded the opposite effect on mental efficiency for low prior knowledge learners (see Figure 2B). Post boc analyses revealed that mental efficiency of note takers was influenced by their level of prior knowledge. More specifically, mental efficiency of note takers with high prior knowledge was higher than for note takers with low prior knowledge $(F(1,55)=21.930$, MSE $=$ $\left.17.408, p<.001, \eta_{\mathrm{p}}^{2}=.285\right)$. Figure 3 provides an overview of the mental efficiencies for each condition.

In addition to the interaction effect, a main effect of prior knowledge on mental efficiency was found during the transfer phase $(F(1,55)=21.077$, MSE $=16.731, p<$ $.001, \eta_{\mathrm{p}}^{2}=.277$ ). The high prior knowledge group revealed a higher mental efficiency while working on transfer tasks than the low prior knowledge group (see Table 1). No other significant effects were found.

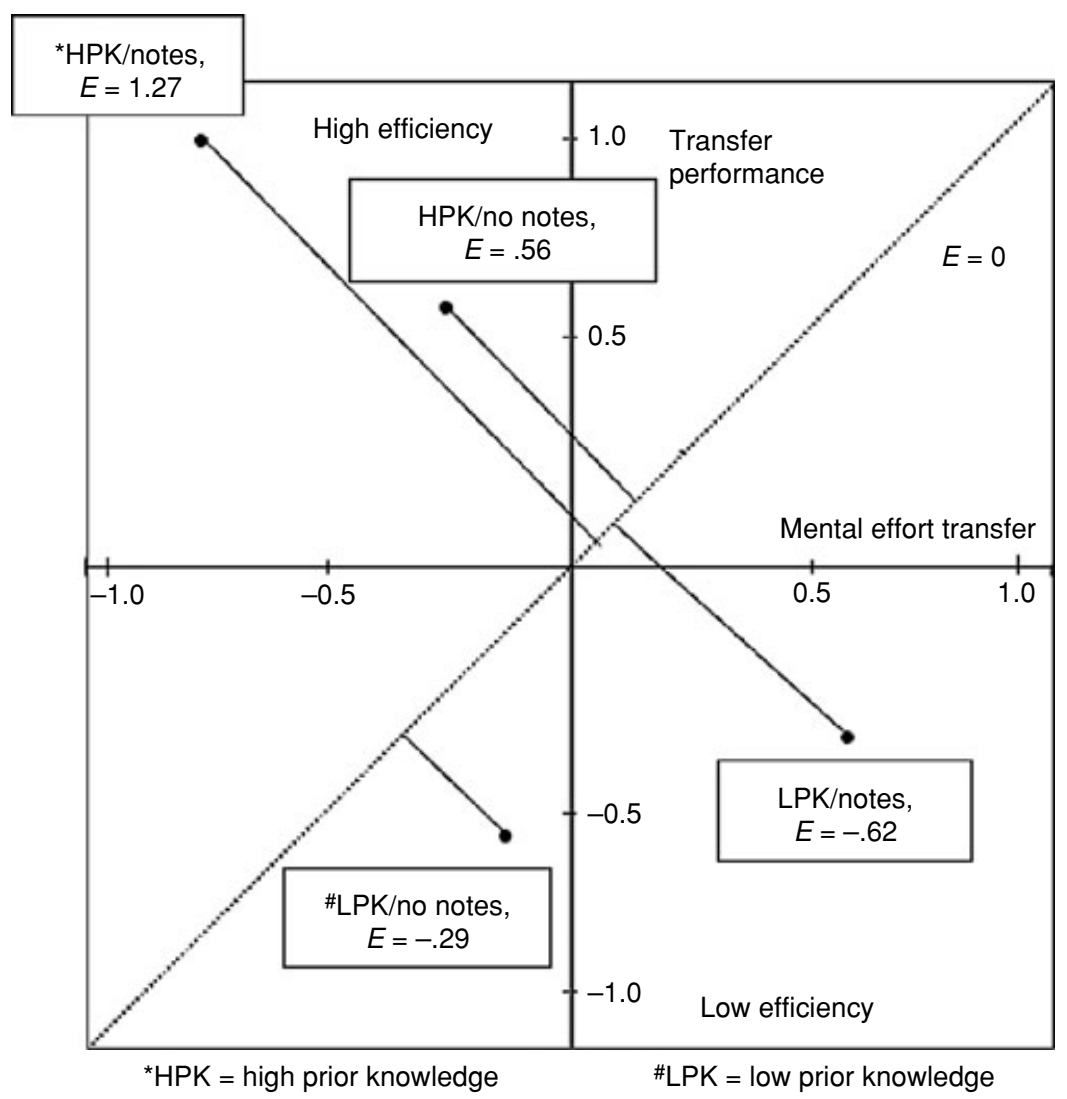

Figure 3. Mental efficiency per condition. 


\section{Activated knowledge}

Table 2 provides the means and standard deviations of the number of concepts, relations between concepts, and correct relations in the notes and the think-aloud protocols.

Table 2. Means and standard deviations of number of concepts, relations between concepts, and correct relations in the notes and think-aloud protocols

\begin{tabular}{|c|c|c|c|c|}
\hline & \multicolumn{2}{|c|}{$\begin{array}{l}\text { Low prior knowledge } \\
\text { group }(N=20)\end{array}$} & \multicolumn{2}{|c|}{$\begin{array}{l}\text { High prior knowledge } \\
\text { group }(N=1 \mathrm{I})\end{array}$} \\
\hline & $M$ & $S D$ & $M$ & $S D$ \\
\hline \multicolumn{5}{|l|}{ Notes } \\
\hline Concepts & 4.30 & 4.56 & 8.18 & 6.56 \\
\hline Relations & 1.55 & 4.57 & 0.45 & 1.04 \\
\hline \multirow[t]{3}{*}{ Correct relations } & 1.30 & 4.01 & 0.27 & 0.65 \\
\hline & \multicolumn{2}{|c|}{$\begin{array}{l}\text { Low prior knowledge } \\
\text { group a }(N=12)\end{array}$} & \multicolumn{2}{|c|}{$\begin{array}{l}\text { High prior knowledge } \\
\text { group }{ }^{a}(N=10)\end{array}$} \\
\hline & $M$ & $S D$ & $M$ & $S D$ \\
\hline \multicolumn{5}{|l|}{ Protocols } \\
\hline Concepts & 7.50 & 4.85 & 15.10 & 7.31 \\
\hline Relations & 2.50 & 2.58 & 9.80 & 5.37 \\
\hline Correct relations & 1.25 & 1.77 & 7.50 & 5.02 \\
\hline
\end{tabular}

${ }^{a}$ Based on a subset of all participants.

Notes

A significant difference in number of concepts was found $(t(29)=-1.940, p<.05$, $d=0.68)$. The high prior knowledge group generated significantly more concepts in the notes than the low prior knowledge group. However, both groups did not differ in the number of (correct) relations between activated concepts. The number of relations described in the notes was very low $(M=1.16, S D=3.73)$. Interestingly, the low prior knowledge group showed a tendency to generate more (correct) relations than the high prior knowledge group. However, this seemed to be influenced by two participants in the low prior knowledge group who generated a relatively large number of relations (i.e., 19 and 9 relations of which, respectively, 17 and 7 were correct).

\section{Protocols}

Significant differences between both prior knowledge groups were found for the number of concepts $(t(20)=-2.975, p<.01, d=1.25)$, relations $(t(20)=-4.181, p<.001$, $d=1.73)$, and correct relations $(t(10.857)=-3.751, p<.01, d=1.66)$. The high prior knowledge group generated more concepts in the think-aloud protocols, more relations between activated concepts, and more correct relations than the low prior knowledge group.

\section{Discussion}

The main goal of this study was to investigate the retrieval-directed function of note taking in prior knowledge activation (i.e., mobilization) for learners with different levels 
of prior knowledge. Results supported the hypothesis that the effectiveness of the retrieval-directed function of note taking is influenced by the amount of prior domain knowledge learners already possess. For learners with relatively high prior knowledge about the circulatory system, note taking lowered mental effort while working on transfer tasks and increased mental efficiency during transfer. For learners with relatively low prior knowledge, note taking yielded the opposite effects on mental effort and mental efficiency during transfer. Post boc analyses showed that both mental effort and mental efficiency of note takers were influenced by their level of prior knowledge. More specifically, the invested mental effort of note takers with low prior knowledge was higher than for note takers with high prior knowledge. In addition, the mental efficiency was higher for note takers with high prior knowledge than for note takers with low prior knowledge. Post boc analyses also showed that note taking increased the invested mental effort while working on transfer tasks for low prior knowledge learners; the mental effort of low prior knowledge learners who took notes was higher than the mental effort of low prior knowledge learners who did not take notes. This higher mental effort is unlikely to be an artifact of the requirement to take notes, because it was found in the transfer phase in which note taking was not allowed. In addition, if this difference in mental effort was an artifact of note taking, this effect should have been even stronger for the learning phase that immediately followed prior knowledge activation during which the notes were taken. Furthermore, the lower mental effort as a result of note taking for high prior knowledge learners also refutes the assumption that high school students always report more effort if they are required to do more.

By externally representing their prior knowledge by means of taking notes, learners with high prior knowledge in a domain are enabled to activate concepts and relate these concepts to one another without having to keep them active in working memory. This retrieval-directed function of note taking reduces the load imposed on working memory and prevents learners from being overloaded by activating prior knowledge. The offloading effect of taking notes facilitates the activation process which enhances learning for high prior knowledge learners. Although high prior knowledge learners who took notes showed a lower mental effort and a higher mental efficiency than high prior knowledge learners who were not allowed to take notes, this difference did not reach statistical significance. This might be the result of the small sample size; there were only 11 high prior knowledge learners who took notes and 13 high prior knowledge learners who did not take notes. This explanation is supported by investigating the effect sizes. For mental effort, $\eta_{p}^{2}$ was .037 and for mental efficiency, it was .042. These effect sizes indicate a small to medium effect of note taking lowering mental effort and enhancing mental efficiency for high prior knowledge learners. Replicating this study with a larger number of high prior knowledge learners might provide stronger evidence for the hypothesis that retrieval-directed note taking has beneficial effects for learners with sufficient prior domain knowledge.

The results supported the notion that retrieval-directed note taking in prior knowledge activation has no beneficial effects for learners with only limited prior knowledge about the circulatory system. Performance and mental efficiency did not differ depending on whether or not low prior knowledge learners were allowed to take notes while mobilizing. Note taking even had a negative effect on experienced mental effort; learners with limited prior knowledge who took notes showed a higher mental effort than low prior knowledge learners who did not take notes. If prior knowledge is very limited, learners might not be able to distinguish relevant from irrelevant concepts or draw relations between activated concepts. This makes it difficult for them to build a coherent 
external representation of their prior knowledge by taking notes. Therefore, note taking might not have had any offloading effects on working memory for low prior knowledge learners.

When looking closely at the activated prior knowledge, it was shown that high prior knowledge learners generated more concepts, more relations, and more correct relations in the think-aloud protocols than low prior knowledge learners. They also generated more concepts in their notes than low prior knowledge learners. However, both groups did not differ in the number of (correct) relations between activated concepts. There was even a tendency for low prior knowledge learners to generate more (correct) relations in their notes. This was influenced by two low prior knowledge learners who generated many (correct) relations. These learners might have been more skilled in taking notes. Interestingly, one of these learners showed a transfer test performance that was higher than the mean of the high prior knowledge group that took notes (i.e., 7.01 vs. 6.60). This provides support for the assumption that the quality of notes may influence the learning gains. It is not clear why these two learners were able to generate a relatively large number of relations, but the possibility that they studied between the prior knowledge test and the actual experiment cannot be excluded. In general, the number of relations in the notes was very low for both high prior knowledge and low prior knowledge learners. This is consistent with Kiewra (1985) who found that relational note taking is difficult for learners.

A practical implication that follows from this study is that retrieval-directed note taking does not necessarily support the activation process and learning. In contrast to traditional note taking research that has found abundant evidence for the encoding and external storage functions of note taking (Kiewra, 1985; Kobayashi, 2005), there is no evidence that the retrieval-directed function of note taking is beneficial in and of itself. Although learners who took notes while activating their prior knowledge performed somewhat higher than learners who did not take notes, this difference did not reach statistical significance. In addition, learners did not differ in invested mental effort or mental efficiency depending on whether they were allowed to take notes or not. The beneficial effect of retrieval-directed note taking seemed to be beneficial for learners who already possessed sufficient prior knowledge. For learners who possessed only limited relevant prior knowledge, it was not beneficial to take notes while activating prior knowledge. For them, note taking resulted in an increase in invested mental effort while working on tasks, indicating that they might be overloaded by the process of taking notes. Therefore, when instructing students to take notes while activating their prior knowledge, teachers should take their students' levels of prior knowledge into account. In addition, instructing students on how to take notes might be beneficial for learning. The analysis of the notes showed that learners did not generate many relations in their notes. If students are encouraged to establish relationships in their notes, this may strengthen the organizing framework facilitating the integration and thus learning of new information.

In the present study, all participants were high school students. Therefore, it is reasonable to conclude that both high and low prior knowledge learners were on the low end of the expertise continuum. Medical students, for example, are considered to have much more elaborated and refined prior knowledge or expertise about the circulatory system than high school students. These learners with relatively high expertise might not benefit from taking notes while activating prior knowledge in the same way as the more knowledgeable high school students in this study do. It is possible that the positive effects of note taking fade away or reverse with increasing expertise (the so-called 
'expertise reversal effect', see Kalyuga, Ayres, Chandler, \& Sweller, 2003). Learners with expertise in a certain domain may already possess a coherent representation of their prior knowledge that can be held in working memory without overloading it. Therefore, note taking might not have any offloading effects for these high-expertise learners. Future research should thus extend the continuum of prior knowledge towards higher levels of expertise and investigate the effects of retrieval-directed note taking in prior knowledge activation for learners at different points of this continuum.

Another line of research is related to the influence of the type of representation used to guide prior knowledge activation. In this study, learners are provided with a static picture that might support them in activating their prior knowledge as compared to not being provided with this picture. But what happens if learners are provided with an animation? Is an animation better able to represent and activate a dynamic domain, such as the circulatory system, as compared to a static picture? And how is the effectiveness of the representation influenced by learners' prior knowledge? Future research may shed more light on this issue.

Concluding, the present study provided evidence that retrieval-directed note taking may exert positive effects on learning. However, these beneficial effects were influenced by the amount of prior knowledge learners already possessed, and were restricted to measures of mental effort and mental efficiency. Future research might provide more insights into the beneficial effects of retrieval-directed note taking in prior knowledge activation.

\section{References}

Anderson, J. R. (1983). The architecture of cognition. Cambridge, MA: Harvard University Press. Anderson, R. C. (1977). The notion of schemata and the educational enterprise: General discussion of the conference. In R. C. Anderson, R. J. Spiro, \& W. E. Montague (Eds.), Schooling and the acquisition of knowledge (pp. 415-431). Hillsdale, NJ: Erlbaum.

Austin, J. L., Gilbert Lee, M., Thibeault, M. D., Carr, J. E., \& Bailey, J. S. (2002). Effects of guided notes on university students' responding and recall of information. Journal of Behavioral Education, 11, 243-254. doi: 10.1023/A:1021110922552

Baddeley, A. D. (1992). Working memory. Science, 255, 556-559. doi: 10.1126/science. 1736359

Barnett, J. E., Di Vesta, F. J., \& Rogozinski, J. T. (1981). What is learned in note taking?. Journal of Educational Psychology, 73, 181-192. doi: 10.1037/0022-0663.73.2.181

Chi, M. T. H., de Leeuw, N., Chiu, M., \& LaVancher, C. (1994). Eliciting self-explanations improves understanding. Cognitive Science, 18, 439-477.

De Grave, W. S., Schmidt, H. G., \& Boshuizen, H. P. A. (2001). Effects of problem-based discussion on studying a subsequent text: A randomized trial among first year medical students. Instructional Science, 29, 33-44. doi: 10.1023/A:1026571615672

Di Vesta, F. J., \& Gray, G. S. (1972). Listening and notetaking. Journal of Educational Psychology, 63, 8-14. doi: 10.1037/h0032243

Goetz, E. T., Schallert, D. L., Reynolds, R. E., \& Radin, D. I. (1983). Reading in perspective: What real cops and pretend burglars look for in a story. Journal of Educational Psychology, 75, 500-510. doi: 10.1037/0022-0663.75.4.500

Kalyuga, S., Ayres, P., Chandler, P., \& Sweller, J. (2003). The expertise reversal effect. Educational Psychologist, 38, 23-31. doi: 10.1207/S15326985EP3801_4

Kiewra, K. A. (1985). Investigating notetaking and review: A depth of processing alternative. Educational Psychologist, 20, 23-32. doi: 10.1207/s15326985ep2001_4

Kiewra, K. A., DuBois, N. F., Christian, D., McShane, A., Meyerhoffer, M., \& Roskelley, D. (1991). Note taking functions and techniques. Journal of Educational Psychology, 83, 240-245. doi: 10.1037/0022-0663.83.2.240 
Kobayashi, K. (2005). What limits the encoding effect of note taking? A meta-analytic examination. Contemporary Educational Psychology, 30, 242-262. doi: 10.1016/j.cedpsych.2004.10.001

Kobayashi, K. (2009). Comprehension of relations among controversial texts: Effects of external strategy use. Instructional Science, 37, 311-324. doi: 10.1007/s11251-007-9041-6

Machiels-Bongaerts, M., Schmidt, H. G., \& Boshuizen, H. P. A. (1995). The effect of prior knowledge activation on text recall: An investigation of two conflicting hypotheses. British Journal of Educational Psychology, 65, 409-423.

Mayer, R. E. (1979). Twenty years of research on advance organizers: Assimilation theory is still the best predictor of results. Instructional Science, 8, 133-167. doi: 10.1007/BF00117008

Paas, F. (1992). Training strategies for attaining transfer of problem-solving skills in statistics: A cognitive-load approach. Journal of Educational Psychology, 84, 429-434. doi: 10.1037/ 0022-0663.84.4.429

Paas, F., \& van Merriënboer, J. J. G. (1993). The efficiency of instructional conditions: An approach to combine mental effort and performance measures. Human Factors, 35, 737-743.

Peeck, J. (1982). Effects of mobilization of prior knowledge on free recall.Journal of Experimental Psychology: Learning, Memory, and Cognition, 8, 608-612. doi: 10.1037/0278-7393.8.6.608

Peeck, J., van den Bosch, A. B., \& Kreupeling, W. J. (1982). Effect of mobilizing prior knowledge on learning from text. Journal of Educational Psychology, 74, 771-777. doi: 10.1037/0022-0663. 74.5.771

Peper, R. J., \& Mayer, R. E. (1978). Note taking as a generative activity. Journal of Educational Psychology, 70, 514-522. doi: 10.1037/0022-0663.70.4.514

Peper, R. J., \& Mayer, R. E. (1986). Generative effects of note taking during science lectures. Journal of Educational Psychology, 78, 34-38. doi: 10.1037/0022-0663.78.1.34

Schmidt, H. G. (1982). Activation and restructuring of prior knowledge and their effects on text processing. In A. Flammer, \& W. Kintsch (Eds.), Discourse processing (pp. 325-338). Amsterdam: Elsevier North-Holland Publishing.

Slotte, V., \& Lonka, K. (1999). Review and process effects of spontaneous note taking on text comprehension. Contemporary Educational Psychology, 24, 1-20. doi: 10.1006/ceps.1998. 0980

Van Merriënboer, J. J. G., \& Sweller, J. (2005). Cognitive load theory and complex learning: Recent developments and future directions. Educational Psychology Review, 17, 147-177. doi: 10.1007/s10648-005-3951-0

Received 16 October 2008; revised version received 9 June 2010 


\section{Appendix}

\section{Types of completion problems}

Mrs $\mathrm{X}$ has diabetes. After every meal, she injects insulin in the vein of her left upper arm. What way does the insulin travel before it reaches the brain?

Type I

Complete solution steps

I) arm vein

2) superior vena cava

3) right atrium

4) right ventricle

5) lung artery

6) lungs

7)

8)

9)

10)

II)
Type II

Rearrange solution steps

I) superior vena cava

2) aorta

3) left atrium

4) right ventricle

5) lung artery

6) lung vein

7) brain

8) left ventricle

9) lungs

10) right atrium

II) arm vein
Correct solution

I) arm vein

2) superior vena cava

3) right atrium

5) right ventricle

5) lung artery

6) lungs

7) lung vein

8) left atrium

9) left ventricle

10) aorta

II) brain 